\title{
Research Article \\ Description of a 2-Bit Adaptive Sigma-Delta Modulation System with Minimized Idle Tones
}

\author{
E. A. Prosalentis and G. S. Tombras \\ Laboratory of Electronics, Department of Physics, University of Athens, Panepistimiopolis, 15784 Athens, Greece \\ Correspondence should be addressed to G. S. Tombras, gtombras@phys.uoa.gr
}

Received 3 June 2007; Revised 24 September 2007; Accepted 28 October 2007

Recommended by Jiri Jan

A 2-bit adaptive sigma delta modulation system that inherently eliminates the idle tones present in conventional and other adaptive sigma delta systems is described. The system incorporates both memory and look-ahead instantaneous step-size estimations and, as shown by computer simulation results apart from eliminating the unwanted idle tones despite dithering, it offers improved SNR performance and extended dynamic range.

Copyright ( $) 2008$ E. A. Prosalentis and G. S. Tombras. This is an open access article distributed under the Creative Commons Attribution License, which permits unrestricted use, distribution, and reproduction in any medium, provided the original work is properly cited.

\section{INTRODUCTION}

Sigma-delta modulation (SDM) is extensively used in various applications due to its high resolution and relatively simple analog implementation. In a simplifying SDM system analysis, the effect of the corresponding 1-bit quantization is widely approximated by an additive white noise model, although generally the quantization error is not white. Indeed, considering quantization of DC input signals, the resulting waveform can be periodic by revealing the so-called idle tones or a noise pattern. This tonal behavior may cause problems when SDM is particularly used in audio applications. In this respect, various dithering techniques have successfully employed in whitening the pattern noise with different amounts of dynamic range degradation [1-4].

Adaptive sigma-delta modulation (ASDM) is considered as an alternative to SDM offering increased dynamic range and reduced quantization noise at the expense of some added complexity [1]. This is achieved by varying the step-size of the basic two-level quantizer according to a decided rule. Such a rule may include backward and/or forward step-size estimation process and is originated from similar rules as applied in single- or multibit adaptive delta modulation (ADM) schemes due to the well-known relation between delta and sigma-delta modulation: a sigma-delta modulator is a delta modulator that encodes the input signal rather than the input signal itself. A good example of a multibit ASDM that originated from a similar ADM scheme is the 2-bit ASDM system by Aldajani and Sayed $[5,6]$, the quantizer of which follows a forward or look-ahead step-size estimation and generates 2-bit output codewords with information about both the sign and the relative magnitude of the step-size resulting in an exponential step-size variability.

Recently, we have presented a 2-bit ADM system that incorporates both memory (backward) and look-ahead (forward) instantaneous step-size estimatios [7]. The origin of that system was a 2-digit ADM system presented in [8], which has been - to the best of our knowledge-the first multidigit instantaneously ADM system with memory and look-ahead step-size adaptation logic. One of the advantageous features of that system has been its inherent ability to eliminate the periodic pattern that characterized the quantization error of the widely known Jayant's ADM with 1-bit memory $[9,10]$.

Motivated by this particular feature and following the aforesaid relation between delta and sigma-delta modulation, in this paper we propose a 2-bit ASDM system based on our recently presented 2-bit ADM in order to examine its operational characteristics and, particularly, to investigate in tonal behavior, that is, the generation of output idle tones for DC input signals. As shown by computer simulation, the proposed system appears to generate minimum, if not none, idle tones despite dithering while it offers high signalto-noise power ratios (SNRs) and extended dynamic range. 
The rest of the paper is organized as follows. In Section 2, both SDM and ASDM are briefly described with particular emphasis given to the Aldajani and Sayed's old 2-bit ASDM. The proposed new 2-bit ASDM is described in Section 3, while simulation results that show the obtained superior performance of the proposed new system in comparison to SDM and the considered old 2-bit ASDM systems under normalized conditions without and with dithering are given in Section 4. Finally, concluding remarks are given in Section 5.

\section{BRIEF DESCRIPTION OF SDM AND ASDM}

The operation of SDM is based on 1-bit quantization of the output $p(n)$ of a noise shaping filter $H(z)$ generating an output binary signal $y(n)=\operatorname{sign}[p(n)]$ denoted as $L(n)=$ $\operatorname{sign}[p(n)] \cdot \Delta$ with $\Delta$ being the fixed-valued step-size of the quantizer and $L(n)$ the generated 1-bit output codeword. In this respect, the lowband portion of $y(n)$ 's frequency spectrum will contain the input signal, while if $H(z)$ is a simple integrator, then

$$
p(n)=p(n-1)+e(n)
$$

with $p(0)=0$ and $e(n)$ being the error signal at time instant $n$ that results from input sample $x(n)$ after subtracting the binary encoded output signal $y(n)$.

Considering the 2-bit ASDM system described by Aldajani and Sayed, [5], the step-size $\Delta$ of the employed quantizer varies according to the general form common to all instantaneous step-size adaptation algorithms [5-11]:

$$
\Delta(n)=M(n) \Delta(n-1),
$$

where $\Delta(n)$ is the step-size magnitude at time instant $n$ with values within a region $\left[\Delta_{\min }, \Delta_{\max }\right]$, and $M(n)$ is the corresponding step-size multiplier defined as

$$
M(n)= \begin{cases}\alpha & \text { if }|p(n)|>\Delta(n-1), \\ \frac{1}{\alpha} & \text { otherwise }\end{cases}
$$

with $a>1$ [5].

Consequently, the encoded output signal $y(n)$ is written as

$$
y(n)=\operatorname{sign}[p(n)] \cdot \Delta(n),
$$

while the generated 2-bit output codeword consists of a first bit denoted as

$$
L_{1}(n)=\operatorname{sign}[p(n)]
$$

and a second bit defined as

$$
L_{2}(n)= \begin{cases}+1 & \text { if }|p(n)|>\Delta(n-1), \\ -1 & \text { otherwise. }\end{cases}
$$

Hence, the step-size adaptation rule of the considered 2bit ASDM can be expressed in compact form:

$$
\Delta(n)=\alpha^{L_{2}(n)} \Delta(n-1),
$$

and the so encoded output signal in the form

$$
y(n)=L_{1}(n) \cdot \alpha^{L_{2}(n)} \Delta(n-1) .
$$

\section{DESCRIPTION OF THE PROPOSED NEW 2-BIT ASDM SYSTEM}

Based on the relation between delta and sigma-delta modulations, the recently presented 2-bit ADM system in [7] can be easily converted into a 2-bit ASDM scheme by simply moving the integrator from the local feedback path prior the input adder of the 2-bit ADM system just after the adder in the forward path. The result is a new 2-bit ASDM system, which is shown in Figure 1. Moreover, the new ASDM system utilizes both "memory" and "look ahead" characteristics in its stepsize estimation process as its origin and generates output codewords that consist of two bits, $L_{1}(n)$ and $L_{2}(n)$. These bits convey information about both the sign of the encoded signal $y(n)=\operatorname{sign}[p(n)] \cdot \Delta(n)$, that is, $y(n)=L_{1}(n) \cdot \Delta(n)$, and the magnitude of the step-size multiplier $M^{\prime}(n)$ defined as

$$
M^{\prime}(n)=M(n) \cdot \gamma(n)=\alpha^{L_{1}(n) L_{1}(n-1)} \beta^{L_{2}(n)} \gamma(n),
$$

where $M(n), y(n)$ are specified below along with constants $\alpha$ and $\beta$.

In particular, $M(n)$ is determined by

$$
\begin{aligned}
M(n) & =N(n) \cdot \beta^{L_{2}(n)} \\
& = \begin{cases}N(n) \cdot \beta & \text { if }|p(n)| \geq \frac{1}{2}\left(\beta+\frac{1}{\beta}\right) N(n) \Delta(n-1), \\
\frac{N(n)}{\beta} & \text { otherwise, }\end{cases}
\end{aligned}
$$

where $\beta>1$ and

$$
N(n)= \begin{cases}\alpha & \text { if } L_{1}(n)=L_{1}(n-1), \\ \frac{1}{\alpha} & \text { if } L_{1}(n) \neq L_{1}(n-1)\end{cases}
$$

with $\alpha>1$, while

$$
\gamma(n)= \begin{cases}\gamma & \text { if } L_{2}(n)=L_{2}(n-1)=-1, \\ 1 & \text { otherwise, }\end{cases}
$$

where $\gamma>1$.

According to (9)-(12), the estimation of $M^{\prime}(n)$ depends on the magnitude of the output $p(n)$ of the mentioned noise shaping filter $H(z)$ (e.g., an integrator) through (10), as well as on a double "memory" element, one dealing with the relation between $L_{1}(n)$ and $L_{1}(n-1)$ and one with the relation between $L_{2}(n)$ and $L_{2}(n-1)$. Hence, at each time instance $n$, the 2-bit output codeword uniquely specifies one out of six possible values of $M^{\prime}(n)=M(n) \gamma(n)=\Delta(n) / \Delta(n-1)$ to the appropriate demodulator, due to the "memory" characteristics in the step-size estimation process [7].

Finally, the values of $\alpha, \beta$, and $\gamma$ are chosen as follows:

(i) $\alpha$ is a set equal to constant $P$ of the constant factor delta modulator [7-9], that is, $1<\alpha \leq 2$;

(ii) $\beta$ must be greater than $\alpha^{2}$, where the exponent 2 reflects the bit-rate relationship between the described scheme and SDM $[7,8]$;

(iii) $1<\gamma<\beta$ in order to ensure convergence of the encoder [7]. 


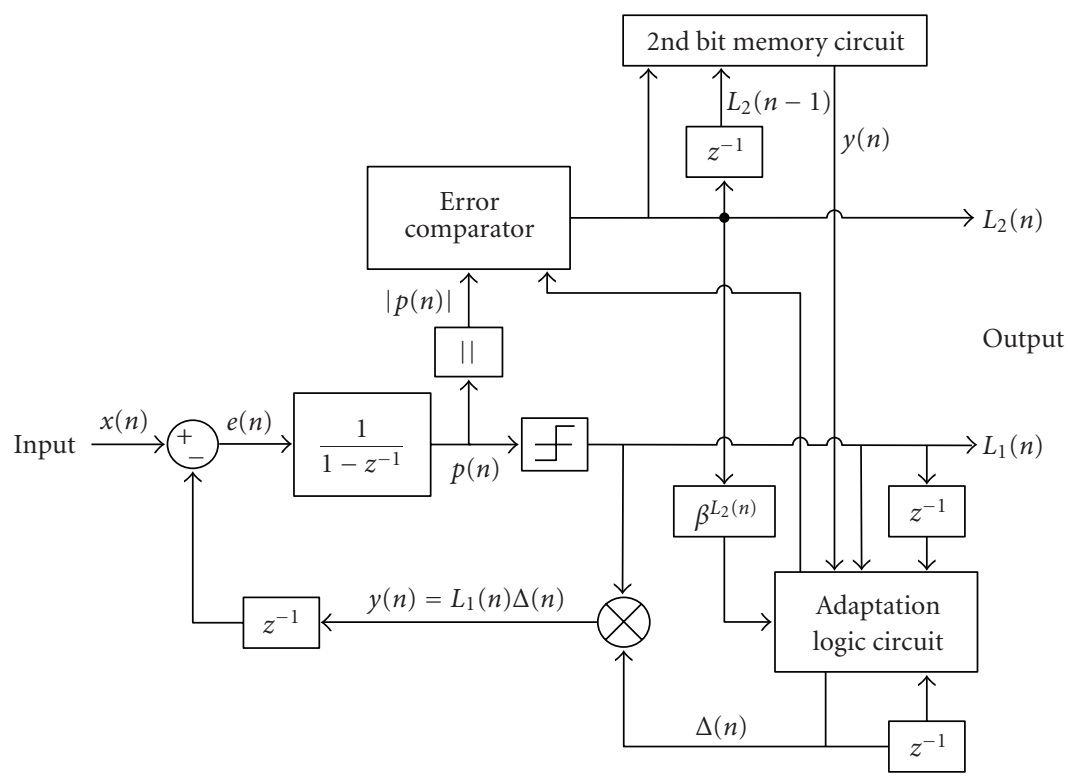

FIGURE 1: Block diagram of the proposed new 2-bit ASDM system.

\section{SIMULATION RESULTS}

In this section, we present computer simulation results comparing the performance of the described new 2-bit ASDM system to that of SDM and the previously considered old 2bit ASDM system.

At first, we use a $20 \mathrm{kHz}$ sine wave input signal with $0 \mathrm{~dB}$ amplitude set at 1 Volt, sampled at $10.24 \mathrm{MHz}$ and ranging from $-120 \mathrm{~dB}$ to $+20 \mathrm{~dB}$. All systems are considered to generate the same output bit rate, meaning that the two 2-bit ASDM systems operate at $5.12 \mathrm{MHz}$. In addition, for both ASDM systems, we choose the initial step-size to be $1 \mathrm{mV}$ and the range of its variation equal to $80 \mathrm{~dB}$, that is, $0.5 \mathrm{mV}$ to $5 \mathrm{~V}$, respectively, while for SDM the loop feedback levels are \pm 1 . Finally, for the described new system we choose $\alpha=1.1, \beta=$ $1.75, \gamma=1.15$ while for the old 2-bit ASDM system $\alpha=1.45$. All these values are considered optimum for the chosen type of input signal $[5,7]$.

The comparison is carried out in terms of the achieved SNR for different amplitudes of the sine wave input signal, and the obtained simulation results are shown in Figure 2. The best SNR values are achieved by the SDM system at the expense of a limited input dynamic range. The peak SNR value is given by the linear model definition $[1,3,4]$ and is equal to $68.83 \mathrm{~dB}$, which is in good agreement with the experimental results. The proposed system appears to retain high SNR values in a smoother manner than that of the old 2-bit ASDM, offering stable operation for a wide range of input signal amplitudes.

In a second comparison, we use a DC input signal with amplitude $1 / 256$ volts $(-48.16 \mathrm{~dB})$ sampled at $1.024 \mathrm{MHz}$ in order to compare the tonal behavior of the three systems. For this, the power spectrum and the short-term autocorrelation of the quantization error of each system are estimated, since a simple spectral analysis alone is not sufficient to reveal idle tones that are short-term periodic in time domain [1]. In

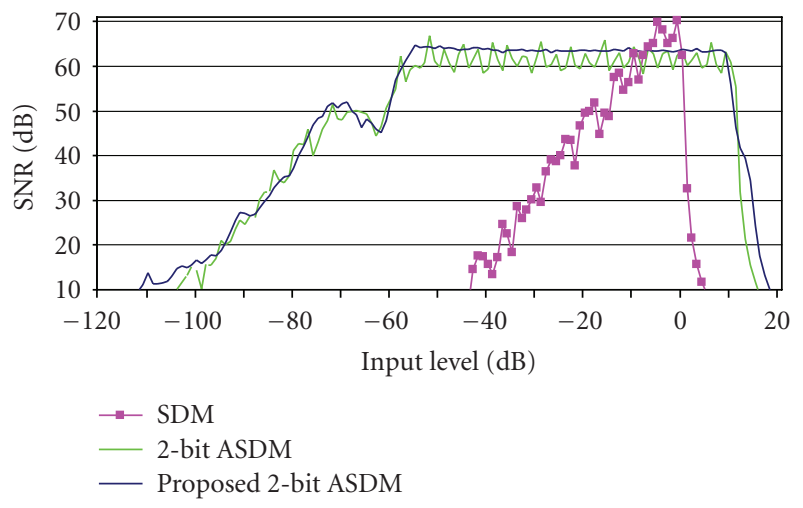

FIGURE 2: SNR versus $20 \mathrm{kHz}$ sine wave input level for the same output bit rate.

spectral calculations, we use a binary output sequence of $2^{20}$ samples and a Blackman-squared window is applied in the time domain prior to the application of Fourier transform to deal with the nonperiodic nature of SDM output signal $[12,13]$. In addition, we use a pseudorandom signal with rectangular probability density function (RPDF) as dither in order to be added to the quantizer input, with spanning one half the quantizer interval, that is, $\delta / \Delta=0.5$, for SDM, and $\delta / \Delta=0.005$ for the two ASDM systems, since it is known that dither is not useful below these thresholds [1].

Considering the operation of all three systems without dithering, it is shown in Figures 3(a) and 3(b) that both SDM and 2-bit ASDM's power spectrum contains detectable lines at discrete multiples of 2 and $6.4 \mathrm{kHz}$, respectively, while the proposed system appears with white-noise-like power spectrum free of such lines. In addition, in Figure 3(c), both SDM and 2-bit ASDM reveal a tonal behavior with a noise pattern repeated at every 256 and 158 samples, respectively, while there is no noise pattern in the output of 


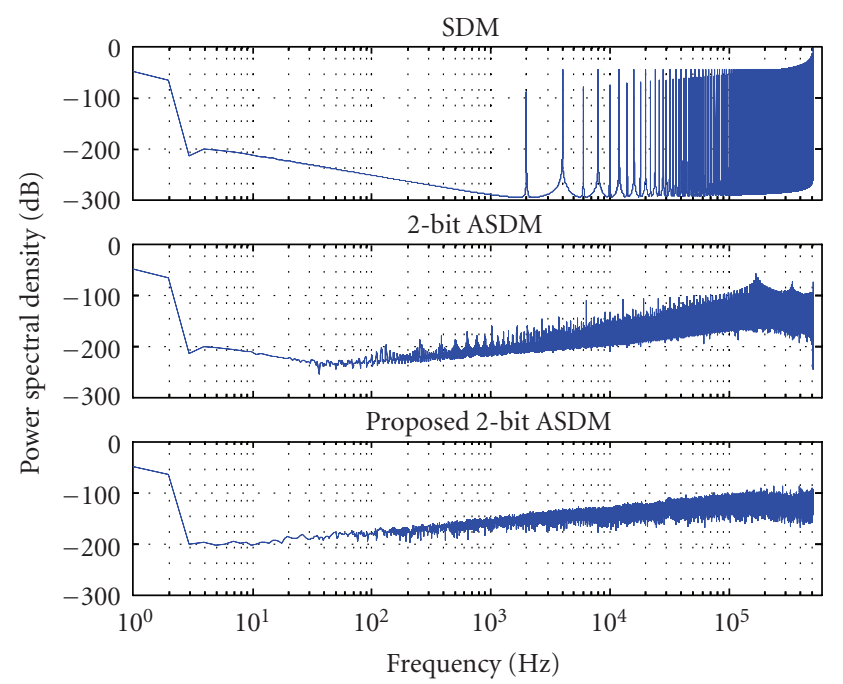

(a)

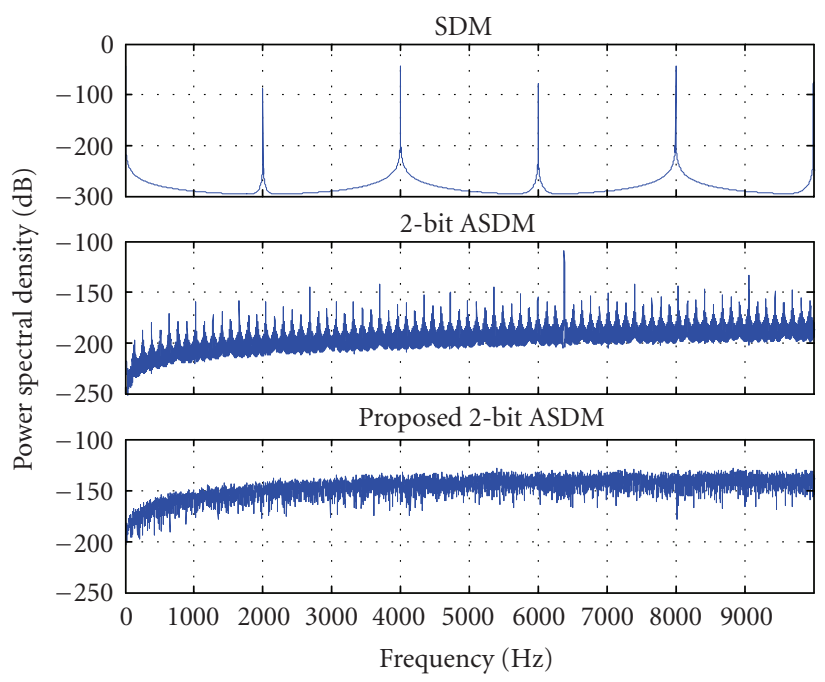

(b)

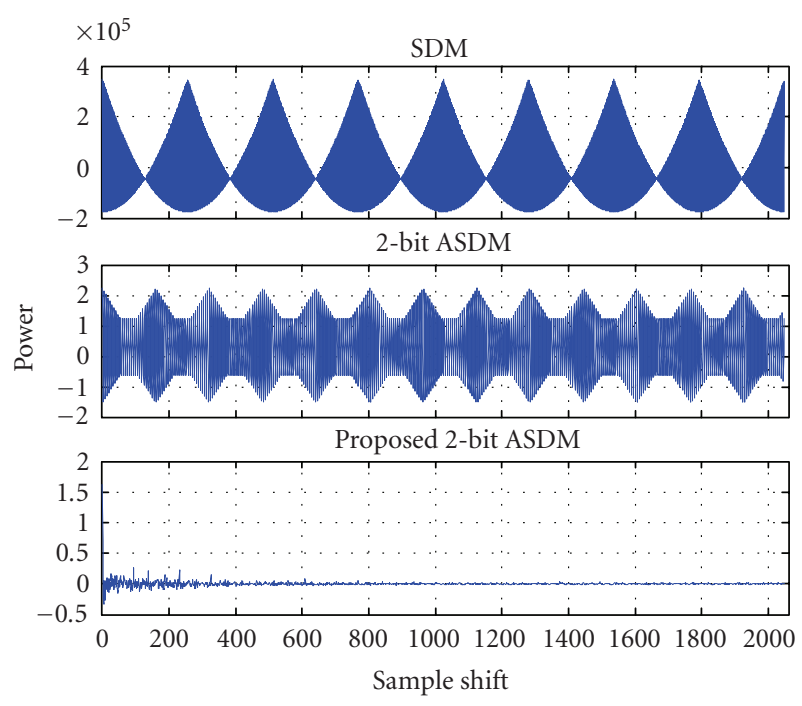

(c)

Figure 3: Performance comparison of the three systems without dithering: (a) full band power spectrum estimation; (b) 0-10 kHz power spectrum estimation; and (c) autocorrelation estimation.

the proposed system. Furthermore, both Figures 3(a) and 3 (b) show that the power spectrum of the proposed 2-bit ASDM (lower graph) follows the spectrum envelope of the 2-bit ASDM (middle graph) except the impulses at the discrete multiples of $6.4 \mathrm{kHz}$ whose magnitude reach up to almost $-60 \mathrm{~dB}$ at $168 \mathrm{kHz}$ (Figure 3(a)) and the first being at $-110 \mathrm{~dB}$ (Figure 3(b)).

Figure 4 now depicts the effect of dithering. As clearly shown, SDM's power spectrum appears free of idle lines (upper graphs in Figures 4(a) and 4(b)), but the autocorrelation estimation reveals again a tonal behavior with a noise pattern repeated at every 256 samples (Figure 4(c)). Similarly, the 2-bit ASDM's power spectrum is also free of idle tones (middle graphs in Figures 4(a) and 4(b)), but although the periodic modulation effect is vanished from its autocorrelation estimation (Figure $4(\mathrm{c})$ ), the baseband noise is almost $50 \mathrm{~dB}$ higher than that without dithering shown in the middle graph of Figure 3(b). Finally, the comparison of the lower graphs in Figures 3 and 4, clearly indicate that the proposed new 2-bit ASDM's power spectrum remains almost unchanged with and without dithering, while dithering causes a small improvement in its autocorrelation estimation.

\section{CONCLUSION}

We have described a new 2-bit ASDM system which, in comparison to SDM and other ASDM systems, and apart its stable operation with high SNR values and extended dynamic range, offers practical elimination of the otherwise expected idle tones despite dithering. The mechanism behind this major and advantageous operational characteristic of the proposed system is not profound, since neither the memory nor the look-ahead feature can justify it by itself. However, a plausible explanation may be the combinational feature that 


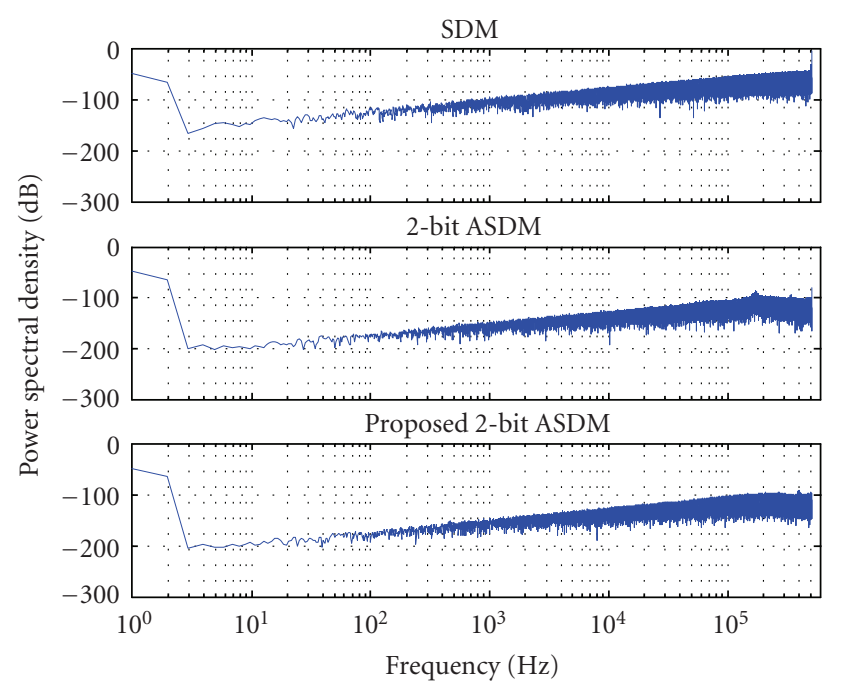

(a)

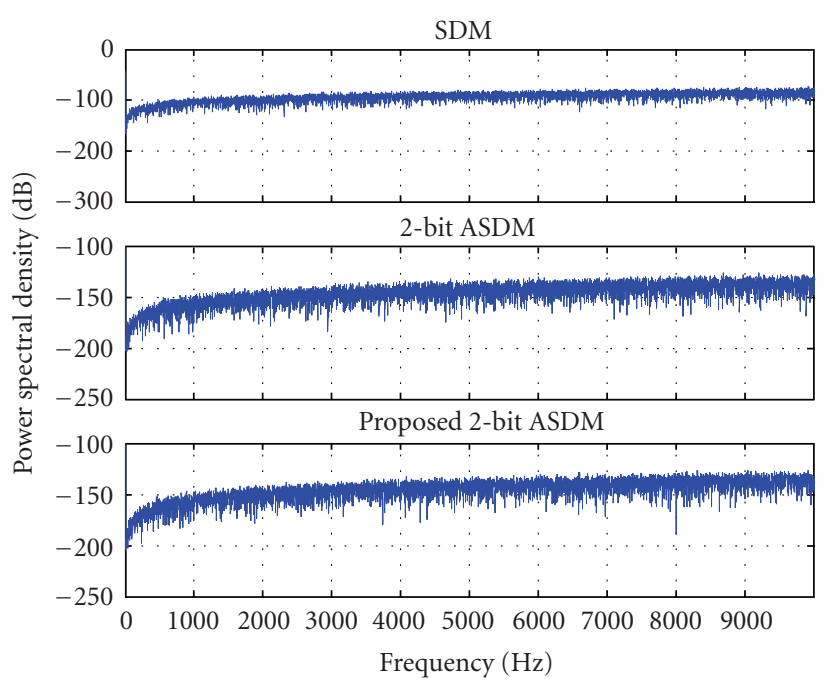

(b)

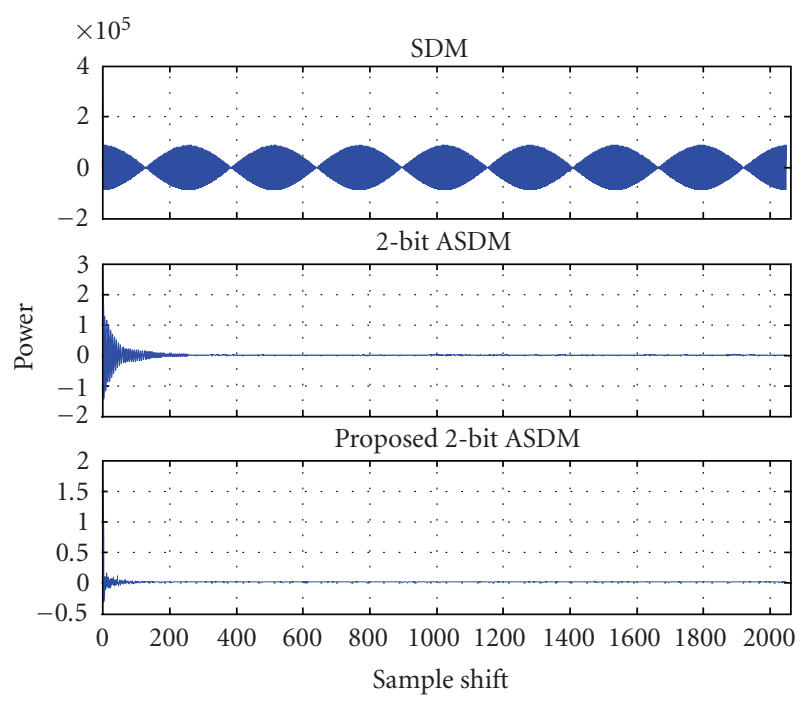

(c)

FIgURe 4: Performance comparison of the three systems with dithering: (a) full band power spectrum estimation; (b) 0-10 kHz power spectrum estimation; and (c) autocorrelation estimation.

inherently exists in the incorporated adaptation logic. In particular, by considering a moderately or a highly varying input signal, there will be a vast number of different step-sizes that will eventually be used during the coding process. Exactly the same seems to be the case for DC input signals. Hence, it is practically impossible to assume that there is a pattern of step-sizes which being used successively gives rise to idle tones as it may be the case for the other two systems under comparison. In any case, the fact that the generation of tonal behavior within the output signal spectrum of the proposed new 2-bit ASDM system is kept minimum if not practically undetectable proves the overall stabilizing influence of both the "memory" and "look-ahead" feature of its step-size adaptation algorithm on its coding process. And this stabilized operation yields enhanced dynamic range, high SNR performance, and robustness in tracking from DC up to highly varying signals, prior the use of any other noise reduction technique.

\section{REFERENCES}

[1] S. R. Norsworthy, R. Schreier, and G. C. Temes, Eds., DeltaSigma Data Converters: Theory, Design, and Simulation, WileyIEEE Press, New York, NY, USA, 1996.

[2] S. R. Norsworthy, "Effective dithering of sigma-delta modulators," in Proceedings of the IEEE International Symposium on Circuits and Systems (ISCAS '92), vol. 3, pp. 1304-1307, San Diego, Calif, USA, May 1992.

[3] X. Sun and K. R. Laker, "Adaptive integrator-output bounding (AIB) for second order sigma-delta ADC," in Proceedings of the 5th International Conference on Signal Processing (ICSP '00), vol. 1, pp. 631-634, Beijing, China, August 2000. 
[4] X. Sun and K. R. Laker, "Tonal behavior analysis of an adaptive second-order sigma-delta modulator," in Proceedings of the IEEE International Symposium on Circuits and Systems (ISCAS '02), vol. 4, pp. 277-280, Phoenix, Ariz, USA, May 2002.

[5] M. A. Aldajani and A. H. Sayed, "A stable adaptive structure for delta modulation with improved performance," in Proceedings of the IEEE International Conference on Acoustics, Speech, and Signal Processing (ICASSP '01), vol. 4, pp. 2621-2624, Salt Lake City, Utah, USA, May 2001.

[6] M. A. Aldajani and A. H. Sayed, "Stability and performance analysis of an adaptive sigma-delta modulator," IEEE Transactions on Circuits and Systems II, vol. 48, no. 3, pp. 233-244, 2001.

[7] E. A. Prosalentis and G. S. Tombras, "A 2-bit adaptive delta modulation system with improved performance," EURASIP Journal on Advances in Signal Processing, vol. 2007, Article ID 16286, 5 pages, 2007.

[8] G. S. Tombras and C. A. Karybakas, "New adaptation algorithm for a two-digit adaptive delta modulation system," International Journal of Electronics, vol. 68, no. 3, pp. 343-349, 1990.

[9] N. S. Jayant, "Adaptive delta modulation with a one-bit memory," The Bell System Technical Journal, vol. 49, no. 3, pp. 321$342,1970$.

[10] N. S. Jayant, "Adaptive quantization with a one-word memory," The Bell System Technical Journal, vol. 52, no. 7, pp. 11191144, 1973.

[11] D. Goodman and A. Gersho, "Theory of an adaptive quantizer," IEEE Transactions on Communications, vol. 22, no. 8 , pp. 1037-1045, 1974.

[12] M. O. J. Hawksford, "Time-quantized frequency modulation with time dispersive codes for the generation of sigma-delta modulation," in Proceedings of the 112th Audio Engineering Soceity Convention (AES '02), Munich, Germany, May 2002.

[13] M. O. J. Hawksford, "Time-quantized frequency modulation, time-domain dither, dispersive codes, and parametrically controlled noise shaping in SDM," Journal of the Audio Engineering Society, vol. 52, no. 6, pp. 587-617, 2004. 